\title{
A propos de narrativité : Interview de Lorenzo Menoud par Nikoleta Kerinska, 2021
}

About narrativity: Interview with Lorenzo Menoud by Nikoleta Kerinska, 2021

\author{
LORENZO MENOUD \\ Écrivain,, artiste, chercheur indépendant - Paris, France
}

NIKOLETA KERINSKA

Université Fédérale d'Uberlândia (UFU) Uberlândia MG, Brésil

\section{RESUME}

Dans cette interview, Lorenzo Menoud, nous propose une réflexion stimulante sur la narrativité. Selon lui, la narrativité n'est pas un concept qui serait défini indubitablement par certaines propriétés essentielles, mais plutôt un terme dont on a tou $\bullet$ te $\bullet$ s une idée, plus ou moins précise et plus ou moins variable. Ainsi, la narrativité se constitue dans un réseau conceptuel au croisement de plusieurs enjeux, parmi lesquels: qu'est-ce que l'on envisage comme un récit et pourquoi ? quels rapports la narrativité entretient-elle avec la fiction et l'art? pour quelles raisons raconte-t-on des histoires? Lorenzo Menoud développe ses idées, en fournissant un cadre théorique essentiel pour comprendre les enjeux du récit et de la narrativité, non seulement dans l'art mais dans une perspective plus générale.

\section{MOTS-CLEFS}

Narrativité, récit, art, image, fiction.

\begin{abstract}
A B S TR ACT
In this interview, Lorenzo Menoud offers us a stimulating reflection on narrativity. According to him, narrativity is not a concept that would be unmistakably defined by certain essential properties, but rather a term of which we all have an idea, more or less precise and more or less variable. Thus, narrativity is constituted in a conceptual network at the crossroads of several issues, including: what is considered a narrative and why? what relationship does narrativity have with fiction and art? for what reasons do we tell stories? Lorenzo Menoud develops his ideas, providing an essential theoretical framework for understanding the issues of narrative and narrativity, not only in art but in a more general perspective.
\end{abstract}

\section{KEYWORDS}

Narrativity, narrative, art, image, fiction. 
Nikoleta Kerinska : Le prochain dossier de la revue État de l'Art s'interroge sur les œuvres contemporaines qui racontent des histoires ou, comme nous allons les appeler, les « œuvres narratives ". Il nous semble que certaines œuvres contemporaines s'engagent sur les voies de la narration au point d'en constituer une thématique à part entière. Selon vous dans quelles conditions peut-on parler de narrativité dans l'art contemporain et plus particulièrement dans les arts visuels ?

Lorenzo Menoud : Il n'y a pas selon moi de narrativité spécifique qui serait propre à l'art contemporain ou aux arts visuels. Il y a des œuvres d'art narratives et des œuvres d'art non narratives, ou des œuvres d'art plus narratives que d'autres, qu'elles soient verbales ou visuelles. En fait, la difficulté d'un concept comme celui de narrativité, c'est qu'il en existe d'innombrables caractérisations. Ainsi, Marie-Laure Ryan, suivant en cela Fotis Jannidis, « suggère de considérer l'ensemble de tous les récits [narratives] comme flou et la narrativité (ou «storyness») comme une propriété scalaire plutôt que comme une caractéristique binaire rigide qui diviserait les représentations mentales en histoires et non-histoires ».

Par ailleurs, il ne paraît pas y avoir de critère décisif pour choisir entre différentes théories narratologiques, Sylvie Patron notant que certaines d'entre elles ne sont pas scientifiques : « II ne me semble pas que l'hypothèse du narrateur fictif dans la narratologie genettienne et dans la narratologie énonciative de Rivara soit une hypothèse falsifiable ». Autrement dit, la narrativité n'est pas un concept qui serait défini indubitablement par certaines propriétés essentielles, mais plutôt un terme dont on a tou•te•s une idée, plus ou moins précise et plus ou moins variable en

fonction du rôle qu'on veut lui faire jouer dans certains contextes spécifiques.

Je trouve donc tout aussi intéressant de constituer un réseau conceptuel où insérer la narrativité que d'en chercher une improbable définition, tant cette idée me semble être au croisement de plusieurs enjeux, parmi lesquels : qu'est-ce que l'on envisage comme un récit et pourquoi ? quels rapports la narrativité entretient-elle avec la fiction et l'art ? pour quelles raisons raconte-t-on des histoires ? Il importe autant d'établir les problématiques dans lesquelles peut s'inscrire le concept de narrativité et de réfléchir à quels pourraient être ses buts que de tenter d'en déterminer la portée et l'usage.

N. K. : Si l'on reprend ces trois questions dans l'ordre dans lequel vous les avez posées : que peut-on considérer comme un récit ? ou comment pourrait-on définir la narrativité ?

L. M. : Avant d'en proposer une caractérisation à proprement parler, j'aimerais au préalable indiquer qu'il faut distinguer deux questions : la controverse narratologique propre à la littérature entre une théorie « communicationnelle » et « non communicationnelle » du récit (SigeYuki Kuroda), d'une part, et l'ouverture ou non de la narrativité à d'autres médias que la littérature, d'autre part, bien que si la posture du•de la narrateur•trice devait ne pas s'avérer nécessaire dans la littérature, comme je le pense, il serait plus facile d'étendre l'idée de récit à d'autres champs artistiques. Autrement dit, il ne faut pas confondre la question de savoir s'il faut ou non un•e narrateur•trice dans tout récit littéraire avec celle qui consiste à déterminer si l'on peut étendre la narrativité au cinéma, à la performance, aux arts numériques, etc. 
Au centre de ces deux controverses, on trouve le point de vue de Gérard Genette qui défend l'idée que tout récit littéraire, à la première comme à la troisième personne, « est, explicitement ou non, « à la première personne», puisque son narrateur peut à tout moment se désigner lui-même par ledit pronom ». En outre, il soutient que l'analyse des récits doit se limiter à la littérature : « Tout récit [...] est une production linguistique assumant la relation d'un ou plusieurs événement(s)».

Depuis longtemps, nombre de théoricien•ne•s ont indiqué en quoi ces conceptions n'étaient pas défendables. Ainsi, dès 1973, Ann Banfield a analysé le discours indirect libre et montré que les récits à la troisième personne ne contiennent pas de narrateur•trice " effacé•e » dans la mesure où les marques subjectives qui s'y trouvent ne peuvent être attribuées à une telle instance.

Quant à l'élargissement de la portée de l'approche narratologique aux autres arts, il était déjà préconisé au sein même du mouvement structuraliste par des auteurs comme Claude Bremond (1964) et Roland Barthes (1966) : «Le récit peut être supporté par le langage articulé, oral ou écrit, par l'image, fixe ou mobile, par le geste et par le mélange ordonné de toutes ces substances ; il est présent dans le mythe, la légende, la fable, le conte, la nouvelle, l'épopée, l'histoire, la tragédie, le drame, la comédie, la pantomime, le tableau peint (que l'on pense à la Sainte-Ursule de Carpaccio), le vitrail, le cinéma, les comics, le fait divers, la conversation ».

\section{N. K. : Quel est votre point de vue sur le sujet ?}

L. M. : J'ai de sérieux doutes quant à la pertinence de la notion de narrateur•trice dans l'analyse de la fiction et des rapports d'énonciation, en particulier dans les récits littéraires à la troisième personne. On prétend habituellement que lae narrateur•trice est censé connaître les lieux et les personnages du récit, alors que l'auteur•trice ne ferait que les imaginer. Il est cependant très curieux de postuler l'existence d'une instance qui aurait connaissance de l'univers de la fiction dans lequel elle est insérée. En effet, imaginer comme certain•e•s théoricien•ne•s que lae narrateur•trice du récit posséderait des informations sur un personnage, qu'iel entretiendrait avec lui un rapport épistémique privilégié ou qu'iel parlerait de l'histoire comme d'un fait connu est injustifié. On ne peut pas attribuer une connaissance directe d'un personnage, c'est-à-dire une perception et une réflexion, à un autre personnage - à moins de postuler que les personnages existent réellement. Que lae narrateur•trice soit souvent supposé•e omniscient•e ou qu'il soit fictionnel qu'iel connaisse le personnage est uniquement une partie du processus de faire-semblant.

On affirme également qu'il y a deux actes différents dans les récits à la première personne. Ainsi, l'acte de Fatima racontant sa vie ne serait pas à confondre avec l'acte de l'autrice Fatima Daas écrivant La petite dernière. Considérer cependant qu'il s'agirait là de deux actes est trompeur : il y a un seul acte narratif, c'est celui de l'auteur trice qui raconte une histoire dans laquelle des personnages réalisent des actions pour de semblant, dont celle, parfois, de raconter une histoire. Mais on comprend aisément en quoi un acte représenté ou un acte fictionnel n'en est pas vraiment un. C'est pourquoi je propose, du moins pour commencer, d'analyser la narration uniquement comme une relation entre des auteur•trice•s et des lecteurs $\bullet$ trice $\bullet s$. Les premier $\bullet$ mière $\bullet s$ inventent des personnages fictifs dont certains occupent un rôle spécifique, mais qui n’a rien 
d'extraordinaire, c'est-à-dire raconter des histoires dans l'histoire racontée par l'auteur•trice. On peut décider d'appeler «narrateur•trice » un tel personnage. Mais présupposer que ce personnage aurait une responsabilité autre qu'en faire-semblant ou qu'il pourrait exister dans une histoire sans pourtant jamais n'y apparaître, comme dans les récits à la 3e personne, c'est oublier le contexte fictionnel initial. Je le répète, il y a un seul acte réel, celui de l'auteur•trice qui raconte, et une quantité d'actes inventés et représentés, ceux des personnages. Certes, l'auteur•trice constitue un personnage comme une personne, ce qui nous oblige à lui donner un statut en faire semblant, à défaut de ne rien y comprendre, mais il reste un personnage, quelque soit son rôle, c'est-à-dire qu'il raconte ou non fictivement l'histoire en question.

Quant à la prétendue distinction entre l'histoire et le récit - c'est-à-dire entre le « contenu narratif » (« signifié ») d'un texte, qui serait modifiable, et « le texte narratif lui-même » (" signifiant »), que l'on ne pourrait altérer -, elle paraît n'avoir aucun fondement. En effet, il n'y a pas d'espace extérieur au texte raconté, celui-ci compose une proposition unique susceptible tantôt d'être résumée, tantôt d'être amplifiée. Évoquer à la manière de la narratologie classique des événements de l'histoire qui seraient omis par le récit, alors tenu pour elliptique, consiste une nouvelle fois à hypostasier un univers fictionnel et à faire comme si des phénomènes s'y déroulaient malgré ce qui nous en est dit.

Enfin, sans la figure intermédiaire d'un•e narrateur•trice qui raconterait, la distinction entre des pratiques artistiques qui disent et celles qui ne feraient que montrer n'est plus pertinente. C'est pourquoi j'estime qu'il faut renoncer à la distinction classique entre le showing et le telling pour simplement distinguer les œuvres narratives de celles qui ne le seraient pas.

\section{N. K. :Alors quelle définition préconiseriez-vous du récit ou de la narrativité ?}

L. M. : Selon le narratologue Gerald Prince : « Un objet est un récit s'il est considéré comme la représentation logiquement cohérente d'au moins deux événements asynchrones qui ne se présupposent ni ne s'impliquent l'un l'autre ». Il affirme que sa définition a plusieurs vertus, en plus du fait qu'elle « n'entre pas en conflit avec les opinions largement répandues sur la nature des récits », dans la mesure où elle " établit une distinction entre les récits et les non-récits (et, plus précisément, entre les récits et la simple représentation d'un événement ou d'une activité, la description d'un processus ou d'un état de choses). Elle distingue également entre les récits et les anti-récits (par exemple, La Jalousie de Robbe-Grillet) en attribuant une cohérence aux premiers ». Bien que Prince réserve sa définition aux récits littéraires, il est possible de l'amender et de l'étendre à toute pratique artistique. Il reste néanmoins plusieurs problèmes à résoudre au préalable.

Tout d'abord, il n'est pas nécessaire d'avoir « au moins deux événements », un seul semblant suffire. Ainsi, l'énoncé : « Elle vécut longtemps » peut être analysé comme un micro-récit. Il s'agit pourtant d'un seul événement, la vie, qui survient à une personne, « elle », que l'on peut découper en plusieurs parties temporelles, comme sa naissance, son enfance, son âge adulte, sa vieillesse et sa mort, à la façon des différents points de la trajectoire d'un objet. Je ne vois pas, par conséquent, en quoi un récit devrait différer de « la simple représentation d'un événement ou d'une activité ». Si la plupart d'entre eux proposent indubitablement des intrigues complexes, 
enchaînant de multiples événements, on ne comprend cependant pas ce qui permettrait d'écarter l'énoncé proposé de la classe des récits.

Je conteste également la cohérence requise par Prince. En effet, une définition est censée rendre compte de ses objets et non les écarter, et ces « anti-récits », qui transgressent les pratiques narratives conventionnelles, sont généralement considérés comme des récits, certes atypiques. Ainsi La Jalousie est explicitement catégorisée comme « roman » sur la première de couverture. En outre,

on a développé de nombreuses reconstructions à même d'accommoder leur structure non linéaire, notamment dans le courant de la « narratologie non naturelle ».

La narrativité de n'importe quel support peut alors se définir, c'est ma proposition, en tant qu'événement représenté. Si l'on reprend rapidement les deux éléments qui constituent cette caractérisation, il s'agit, d'une part, d'événements, c'est-à-dire de phénomènes qui, au contraire des objets, arrivent ou qui se produisent, dont les limites spatiales sont relativement vagues, mais dont les limites temporelles sont plutôt nettes, qui perdurent à travers le temps et que l'on peut découper en séquence ; on a affaire, d'autre part, à des représentations, à savoir à des façons intentionnelles non pas de faire référence au monde, sans quoi la fiction ne pourrait pas être représentationnelle, mais de présenter quelque chose à travers un média, de renvoyer à quelque chose, indépendamment de son existence. En conséquence, je propose le schéma suivant :

fictionnel artistique roman, film, $B D \ldots$

représenté (humain)

artistique poésie documentaire
non fictionnel

non artistique fait divers

- événement

humain construction d'un objet

non représenté

non humain

avalanche

artistique poésie concrète, photo, installation...

représenté (humain)

non artistique article de physique

- non-événement

humain

objet manufacturé

non représenté

non humain

arbre

Figure 1. - Lorenzo Menoud, La narrativité en tant qu'événement représenté, 2021. Source : L. Menoud 
N. K. : Si l'on revient à la deuxième question que vous posiez, quels sont les liens que l'on peut établir entre la narrativité et la fictionalité d'une œuvre ou entre la narrativité et son articité ?

L. M. : La narrativité détermine selon moi la possibilité de la fiction (ou de la non-fiction) d'une œuvre, mais pas son articité (ou non-articité). En effet, une œuvre doit être narrative ou discursive pour être considérée, possiblement, comme fictionnelle. Il faut préalablement qu'une œuvre d'art visuel ou un texte représente un événement pour pouvoir être classé•e (ou non) comme de la fiction, dans la mesure où la simple représentation d'un objet manque du mouvement nécessaire. Un tableau ou une photographie, par exemple, seront alors jugé•e•s comme des représentations fidèles, précises et ressemblantes à leur sujet ou, au contraire, imprécises et maladroites, mais en aucun cas, iels ne pourront être regardé•e•s comme étant référentiel•le•s ou documentaires (ni fictionnel•le•s). Autrement dit, c'est le développement spatio-temporel de la séquence événementielle qui permet à sa représentation d'avoir la multiplicité requise pour être référentielle (ou non). Ainsi, un parcours narratif schématique qui irait de $A$ à $C$, en passant par $B$, pourra alors être envisagé, dans un second temps, soit comme une proposition fictionnelle, plus ou moins inspirée de la réalité, soit comme le compte rendu d'événement(s) réel(s). Les questions qui se poseront afin de déterminer la référentialité ou la fictionalité d'un tel récit étant : ce qui est relaté, $A B C$, prétend-il à la vérité, constitue-t-il une tentative de description de la réalité, d'un événement ou groupe d'événements ayant eu lieu ? ou est-ce, au contraire, une histoire, plus ou moins inventée, qui n'aspire aucunement à énoncer ce qui est le cas, et qui se présente d'emblée comme telle, dans un cadre institutionnel ou social déterminé - contrairement au mensonge qui entend tromper en se faisant passer pour véridique?

Par contre, la narrativité (ou non) d’une séquence événementielle ne joue aucun rôle dans la détermination de l'articité de sa représentation, dans la mesure où il existe un grand nombre d'œuvres d'art fixes, représentant des objets, comme des sculptures ou des installations, qui ne sont pas susceptibles d'être fictionnelles (ni non fictionnelles), c'est-à-dire où cette opposition ne se pose pas. Voici un second schéma, complétant le premier, qui pourrait expliciter les liens de dépendance (inclusion) entre les différentes problématiques : 


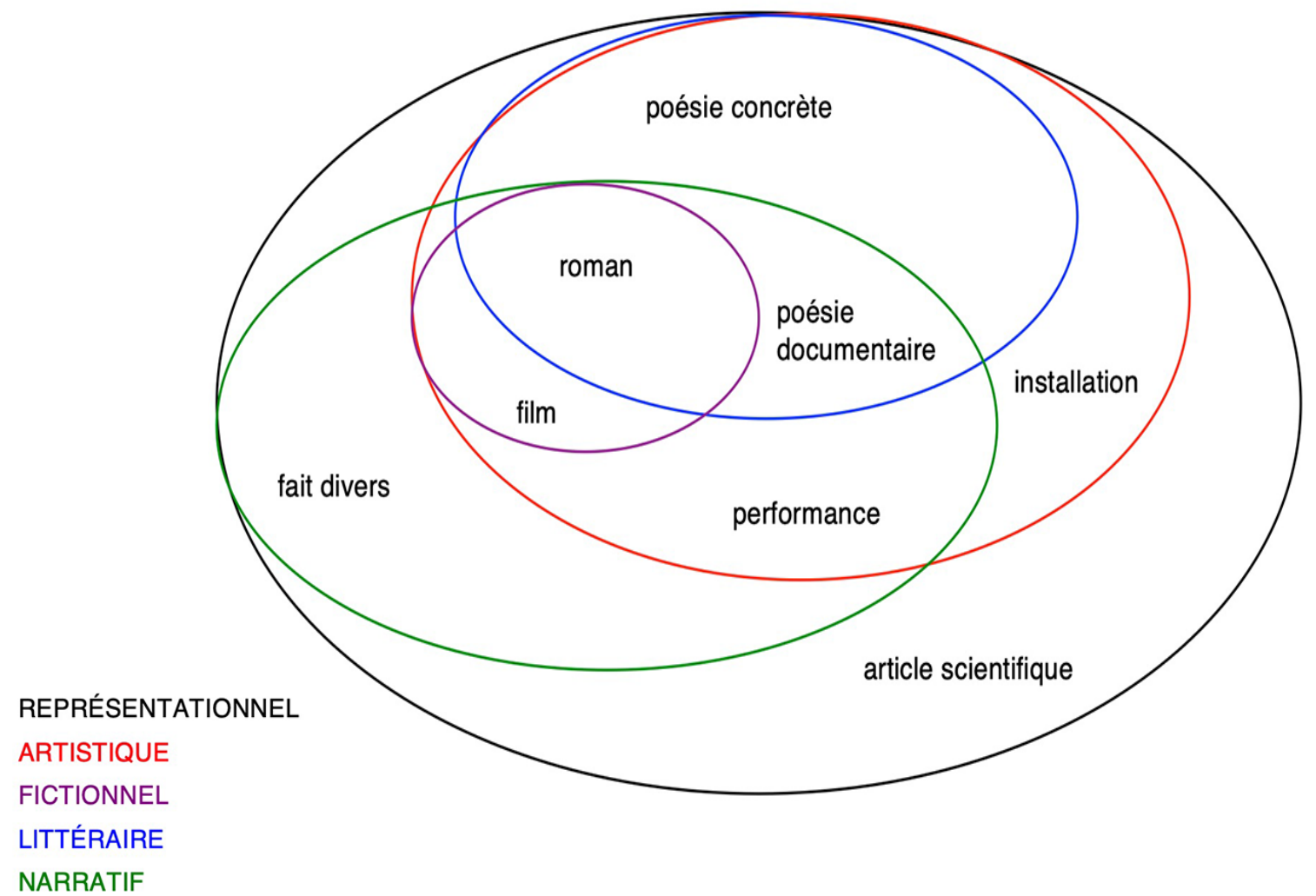

Figure 2. - Lorenzo Menoud, Liens de dépendance entre les différentes problématiques, 2021.

Source: L. Menoud.

N. K. : Est-ce qu'un récit peut alors être composé uniquement d'images ? Autrement dit, pensez-vous qu'un récit pourrait se passer du langage naturel ?

L. M. : En vertu de ce qui précède, cela est tout à fait possible. Il y a des exemples notables de récits qui n'emploient pas le langage naturel, ceux du cinéma muet ou de la bande dessinée sans texte notamment. Il existe par ailleurs des images dans lesquelles coexistent plusieurs moments de la vie d'un même individu, comme certain•e•s tableaux synoptiques et photographies superposées (Hans Memling, Duane Michals...), où un même personnage apparaît simultanément dans différentes situations, sur un plan unique organisé spatialement. Je propose de considérer de telles représentations comme si elles étaient composées d'une série d'images, puisqu'elles possèdent des parties spatiales que l'on pourrait découper et analyser de la sorte - le fait que le même personnage s'y trouve représenté à plusieurs reprises nous incitant à les voir ainsi. Malgré leur apparence, ce ne sont donc pas des images singulières, mais plutôt une multiplicité d'images conjointes qui peuvent être narratives comme n'importe quelle suite d'images.

Cela dit, je me refuse à reconstruire les images fixes en récit en faisant appel, comme certain•e•s théoricien•ne•s, à " l'instant le plus fécond » d'une peinture (Gotthold Ephraim Lessing), ou à des implications qui se trouveraient contenues dans une image en lui plaquant 
des propriétés tirées des objets représentés (Kendall Walton). De tels procédés font uniquement recours à nos capacités encyclopédiques, récognitionnelles ou imaginatives. Une image fixe ne raconte aucune histoire (ou toutes les histoires). En effet, soit un récit (connu) précède l'image en question (mythologie...), et celle-ci n'en est que l'illustration partielle sans qu'elle ne raconte rien, soit on se trouve sans repères devant une telle image et on ne peut qu'imaginer différents parcours de lectures. Et même si l'on admettait qu'une photographie pût être discursive, les histoires qu'une image raconterait dépendraient de celui qui la regarde, ce qui n'est pas le cas - ou dans une très moindre mesure - pour un film ou un livre. C'est pourquoi une seule image fixe ne peut pas être narrative et, partant, fictionnelle (ou non fictionnelle).

Plus généralement, je pense que dans notre interprétation des œuvres d'art et de littérature, il nous faut restreindre au maximum les analogies tirées du monde réel. Non seulement une telle limitation empêche un certain nombre d'inférences délirantes (en admettant que les personnages d'une peinture aient du sang dans les veines, comme le pense Walton, ils devraient alors avoir ou non le virus du Covid-19), mais surtout elle nous permet d'apprécier une œuvre en tant que telle. Si la réalité servait de modèle pour combler les inévitables lacunes d'un récit fictionnel nécessairement incomplet et que d'innombrables caractéristiques étaient injectées à un personnage parcimonieusement spécifié dans l'œuvre en question, ce qui ferait la différence entre deux êtres fictionnels serait purement anecdotique, la majorité et l'essentiel de leurs traits coïncidant. Au contraire, le fait de limiter les propriétés d'un lieu ou d'un personnage à ce qui en est énoncé dans le récit nous permet d'en dégager le principal et d'en comprendre de cette façon les enjeux. Autrement dit, étant donné les limitations auxquelles un•e auteur•trice doit nécessairement faire face dans la création de son univers, il est légitime de penser que les traits choisis (retenus) sont importants et nous guident à porter notre attention sur des éléments significatifs de l'œuvre en question.

\section{N. K. : Vous vous demandiez, c'était votre troisième question, pourquoi raconter des}

\section{histoires ?}

L. M. : C'est une question délicate, à laquelle il est difficile de répondre. J'ai l'impression, à priori, sans donc avoir mené ni lu d'études empiriques sur le sujet, que c'est une pratique universelle. En effet, je ne vois pas comment il serait possible pour une société d'êtres humains de se passer d'histoires, référentielles (ou fictionnelles), dans la mesure où elles leur permettent d'échanger entre eux et de comprendre le monde qui les entoure.

Les histoires peuvent avoir plusieurs fonctions, elles sont en particulier susceptibles de divertir, d'instruire et/ou de transformer les personnes qui les écoutent ou les lisent. Un récit peut être distrayant, par le plaisir qu'il procure à ses auditeur•trice•s ou lecteur•trice•s ; c'est le but visé par un grand nombre de fictions notamment. Il a également des vertus cognitives, il nous donne des informations sur notre environnement, offre une compréhension de phénomènes naturels et humains, voire une certaine maîtrise des événements à travers la narration qui est faite d'une histoire passée ; c'est le cas, par exemple, de la série télévisée The Wire (2002-2008) qui à travers différentes saisons aborde fictionnellement de nombreux problèmes sociaux dans une ville des 
États-Unis (drogue, violences policières, éducation, etc.). Enfin, il arrive qu'un récit soit subversif, qu'il modifie notre être et nos actions sur le monde et autrui ; je pense ici à des performances comme The Mythic Being (1973-1975) d'Adrian Piper dont les actions représentées questionnent l'identité sexuelle et raciale, et peuvent engendrer conséquemment des actions réelles pour lutter contre les discriminations. C'est pourquoi la narrativité occupe une fonction centrale, tant psychologique, cognitive, sociale que politique.

Si l'on considère plus précisément les œuvres narratives artistiques et littéraires, on remarque qu'elles bénéficient d'une grande liberté, puisqu'aucune histoire ne préexiste à la présentation qui en est faite par leur auteur •trice, je l'ai dit, bien qu'elles puissent être inspirées de faits réels et qu'elles soient nécessairement tributaires du monde dans lequel elles se racontent : elles présentent le plus souvent des personnages humains qui s'inspirent des comportements de véritables personnes, les lieux dans lesquels ils se déplacent ressemblent aux lieux que nous occupons sur Terre, le temps passe généralement de la même façon pour eux que pour les êtres réellement existants dont ils empruntent l'essentiel des propriétés, etc. Il est par conséquent plus commode pour les artistes et les écrivain•e•s de fiction de s'émanciper d'une réalité dont iels n'ont pas à rendre compte et de pouvoir déterminer ainsi à volonté le champ de leur pratique. Ce peut être une raison du choix de la fictionalité narrative, au risque cependant d'éloigner ces pratiques d'un certain rapport à l'actualité.

N. K. : Dans une perspective historique, la dimension narrative d'une œuvre peut être envisagée par rapport à la représentation et son activation par la figuration. Si l'on admet que les avant-gardes artistiques du début du XXème siècle ont condamné ces « principes », comment peuton envisager la dimension narrative d'une œuvre contemporaine ?

L. M. : Je pense que la situation a radicalement changé au cours de ces dernières années. La révolution telle que les avant-gardes historiques la concevaient, principalement par le renouvellement vertical des codes expressifs, est désormais dépassée, elle appartient à l'ancien monde. Il me semble qu'une partie toujours croissante des artistes, conscient•e•s des inégalités sociales, considère actuellement

que l'émancipation commence dans le quotidien, dans la subversion des rapports interpersonnels de domination, dans la lutte contre les discriminations structurelles en fonction du genre, de la race, de la religion, etc. C'est pourquoi l'idée d'une avant-garde qui dicterait un agenda esthétique et politique à une population qui devrait être guidée ne fait plus beaucoup de sens aujourd'hui - et c'est tant mieux, tant elle paraît infantilisante et paternaliste. Désormais, j'ai l'impression que les histoires naissent autrement, que les aspirations sont différentes, la fiction pouvant alors parfois sembler loin de nos préoccupations, on a besoin d'implication personnelle, de témoignages, j'en veux pour preuve l'essor depuis quelques années de ce qu'on appelle « l'art documentaire » ou « l'esthétique documentaire ». En quelques mots, ces termes servent à qualifier des pratiques « représentationnelles, non fictionnelles et pleinement artistiques » qui génèrent des documents ou s'emparent d'archives préexistantes afin de reconfigurer notre rapport au réel. Un tel déplacement de perspective est aussi perceptible en théorie du récit avec la narratologie 
dite "postclassique ». Ce courant qui s'est développé à partir de la fin des années 90 questionne certains dogmes structuralistes et propose notamment des narratologies féministe, queer et postcoloniale.

En conclusion, il émerge une génération qui fait le constat de l'inégalité massive régnant dans nos sociétés, et qui privilégie des pratiques artistiques et théoriques pouvant répondre, plus ou moins directement, à la violence faite aux corps et aux esprits des dominé•e•s. Autrement dit, on s'est massivement rendu compte, au-delà du classisme traditionnel, de la prédominance du sexisme, du racisme et de l'islamophobie qui traversent l'ensemble des sociétés occidentales et qui ne sont pas sans affecter les représentations artistiques et intellectuelles. Non seulement les artistes et les théoricien•ne•s abordent de nouvelles problématiques dans leurs travaux, mais iels commencent à modifier le canon en incluant désormais les œuvres et les recherches de celleux dont on avait systématiquement écarté la parole : les femmes et les personnes racisées. C'est ce monde-là - fait de luttes quotidiennes, souvent violentes tant les forces qui pèsent sur les personnes discriminées sont implacables, d'une grande réflexivité, le questionnement et la remise en cause sont permanent•e•s, auquel je n'appartiens pas "naturellement », dans le sens où je ne suis pas de la génération qui l'a vu naître - que je vois actuellement grandir avec beaucoup d'espoirs et de tendresse. 


\section{BIBLIOGRAPHIE suggérée par L. Menoud}

BANFIELD, Ann. "Le style narratif et la grammaire des discours direct et indirect", Change, 16-17, 1973.

BARTHES, Roland. «Introduction à l'analyse structurale des récits », Communications, 8, 1966. https://doi.org/10.3406/comm.1966.1113

BREMOND, Claude. "Le message narratif », Communications, 4, 1964. https://doi.org/10.3406/ comm.1964.1025

BRÜTSCH, Matthias. « How to measure narrativity?: notes on some problems with comparing degrees of narrativity across different media », in Emerging vectors of narratology, Berlin, De Gruyter, 2017. https://doi.org/10.1515/9783110555158-016

BUTLER, Cornelia, CHERIX, Christophe et PLATZKER, David (eds.). Adrian Piper: A synthesis of intuitions 1965-2016, New York, MoMa, 2018.

CAILLET, Aline et POUILLAUDE, Frédéric (dir.), Un art documentaire. Enjeux esthétiques, politiques et éthiques, Rennes, Presses Universitaires de Rennes, 2017.

CASATI, Roberto et VARZI, Achille. «Events», The Stanford Encyclopedia of Philosophy (Summer 2020 Edition), Edward N. Zalta (ed.), https://plato.stanford.edu/archives/sum2020/entries/events/.

DAAS, Fatima. La petite dernière, Paris, Les Éditions Noir sur Blanc, 2020.

GENETTE, Gérard. Figures III, Paris, Seuil, 1972.

GENETTE, Gérard. Nouveau discours du récit, Paris, Seuil, 1983. https://doi.org/10.2307/1772332

HERMAN, David, JAHN, Manfred and RYAN, Marie-Laure (eds.). Routledge Encyclopedia of Narrative Theory, Abingdon, Routledge Ltd, 2005.

JAMES, Alison et REIG, Christophe (dir.), Frontières de la non-fiction. Littérature, cinéma, arts, Rennes, Presses Universitaires de Rennes, 2013.https://doi.org/10.4000/books.pur.56119

KURODA, Sige-Yuki, "Réflexions sur les fondements de la théorie de la narration », 1975, dans Pour une théorie poétique de la narration, Paris, Armand Colin, 2012. https://doi.org/10.3917/arco. patro.2012.01

LESSING, Gotthold Ephraim, Laocoon, Paris, Hermann, 1990. 
MENOUD, Lorenzo. Qu'est-ce que la fiction ? Paris, Vrin, 2005.

- «Fiction de la fiction photographique », dans le catalogue de l'exposition Innuendo, Genève, 2010.

- « Versions », dans Fictions \& médias. Intermédialité dans les fictions artistiques, Paris, Éditions de la Sorbonne, 2011.

- «La fiction augmentée. Une analyse de la narration mixte », dans Images et récits. La fiction à l'épreuve de l'intermédialité, Paris, L'Harmattan, 2013.

PATRON, Sylvie. « Sur l'épistémologie de la théorie narrative (narratologie et autres théories du récit de fiction) », Les Temps modernes, décembre 2005. https://doi.org/10.3917/ltm.635.0262

-, « Le narrateur », introduction à Le Narrateur. Introduction à la théorie narrative, Paris, Armand Colin, 2009, https://www.fabula.org/atelier.php?Le_narrateur.

PATRON, Sylvie (dir.). Introduction à la narratologie postclassique. Les nouvelles directions de la recherche sur le récit, Villeneuve d’Ascq, Presses universitaires du Septentrion, 2018. https://doi. org/10.4000/books.septentrion.19105

PRINCE, Gerald. « Narrativehood, Narrativeness, Narrativity, Narratability », in Theorizing Narrativity, Berlin/New York, De Gruyter, 2008.

PRINCE, Gerald, et QIAO Guoqiang. "Narratology as a Discipline », An Interview with Gerald Prince, Diegesis 1, H. 1, 2012, https://www.diegesis.uni-wuppertal.de/index.php/diegesis/article/ view/62/55.

RYAN, Marie-Laure. Avatars of story, Minneapolis/London, University of Minessota Press, 2006.

WALTON, Kendall. Mimesis as Make-Believe: On the Foundations of the Representational Arts, Cambridge/London, Harvard University Press, 1990. 


\section{À propos des auteurs}

Lorenzo Menoud, écrivain, artiste, chercheur indépendant et mâle blanc de plus de 50 ans.

Livres personnels: J'entrerais par 100 pistes, Éd. Dernier télégramme, Limoges, 2017 ; Autoportrait génétique partiel 1/1000000, coll. Utopistes, Genève, MetisPresses, 2009 ; Qu'est-ce que la fiction? coll. chemins philosophiques, Paris, Éd. Vrin, 2005.

SITE : http://www.serialpoet.eu/

Nikoleta Kerinska est diplômée en textiles, vêtements et accessoires du vêtement de l'École nationale des beaux-arts (1995), spécialisation en sérigraphie de l'École nationale des beaux-arts (1996), maîtrise en art et technologie de l'image de l'Université de Brasilia (2000), et Docteur en Arts Plastiques, Esthétique et Sciences de l'Art de l'Université de Paris I, Panthéon-Sorbonne (2014). Elle est actuellement chercheuse au sein de l'équipe Fictions \& Interactions de I'Université Paris 1 Panthéon-Sorbonne sous la coordination de Bernard Guelton. Depuis 2003, elle est professeur d'art informatique à l'Université fédérale d'Uberlândia. Ses recherches artistiques s'inspirent des convergences et divergences des processus de communication homme-machine, qui font appel au langage naturel, ainsi que des relations poétiques entre langage naturel et image.

LATTES : http://lattes.cnpq.br/9119298795241795

ORCID : https://orcid.org/oooo-0001-5486-1381

SITE : https://nk.artificialis.org/

Resu en: 9-06-2021

\section{Comment citer}

MENOUD Lorenzo; KERINSKA, Nikoleta (2021). A propos de narrativité : Interview de Lorenzo Menoud par Nikoleta Kerinska, 2021. Revista Estado da Arte, Uberlândia. v.2, n.1, p. 219-231, jan./jun. 2021. https:// doi.10.14393/EdA-v2-n1-2021-61570 